\title{
A Comparative Study of Bronchial Washing and Brushing, with Bronchial Biopsy
}

\author{
Jayakrishnan C. ${ }^{1}$, Kamala V. V. ${ }^{2}$ \\ ${ }^{1}$ Department of Pathology, Government Medical College, Thrissur, Kerala, India. \\ ${ }^{2}$ Department of Pathology, Government Medical College, Thrissur, Kerala, India.
}

\section{ABSTRACT}

\section{BACKGROUND}

Lung cancer is the most common cancer around the world and is associated with significant mortality. Smoking is the most important risk factor. Screening and early diagnosis has significant role in initiating treatment and reducing mortality rates. Also, definite histological subtyping is mandatory for newer therapeutic strategies. Over the past decades, screening for lung cancer has evolved with the advent of bronchoscopy and cytological evaluation. Even though various biomarkers of lung cancer are in study, cytological evaluation of bronchial brushing and washing has become a useful and cost-effective screening method for lung cancer and is widely used all around the world.

\section{METHODS}

It was a comparative cross-sectional study conducted in the Department of Pathology, Government Medical College, Thrissur, from 1-1-2017 to 30-6-2018. Bronchial washing and brushing specimens received along with biopsy, which are suspicious for bronchogenic carcinoma were included in the study. Sample size was 82. Cytological smears are stained with Giemsa staining and Papanicolaou's staining. Biopsy specimens are stained with haematoxylin and eosin staining after processing.

\section{RESULTS}

Majority of patients were in the age group of $60-79$ years (75.6\%), and mean age was 64.14 years. Cigarette smoking was seen in 55 patients $(67.1 \%)$. Out of the 82 cases, 46 cases (56\%) were squamous cell carcinoma, 14 cases (17\%) were adenocarcinoma, 15 cases $(18.3 \%)$ were non-small cell carcinoma- NOS, 7 cases $(8.6 \%)$ were small cell carcinoma. Positivity rates for malignancy with brushing was $52.4 \%$, washing $19.5 \%$ and combined $54.9 \%$. Bronchial brushing had a sensitivity of $19.6 \%$ in squamous cell carcinoma, $28.6 \%$ in adenocarcinoma, $26.7 \%$ in non-small cell carcinoma (NSCLC)-NOS, $28.6 \%$ in small cell carcinoma (SCLC). Compared to bronchial brushing, the sensitivity of bronchial washing was lower and combination of washing with brushing yielded no additive effects except in adenocarcinoma cases.

\section{CONCLUSIONS}

Among routinely employed cytological techniques in our centre for a diagnosis of bronchogenic carcinoma, bronchial brushing has higher sensitivity and specificity than bronchial washing. Even though the combination of both cytological techniques yielded not much difference in sensitivity and specificity than brushing alone, usefulness of bronchial washing was evident in adenocarcinomas. Therefore, bronchial brushing, washing and biopsy should be done in the evaluation of suspicious bronchogenic carcinoma and major importance in the processing, evaluation and analysis of brushing will be useful in better cytological diagnosis.

\section{KEY WORDS}

Lung Cancer, Bronchial Brushing, Bronchial Washing, Squamous Cell Carcinoma, Adenocarcinoma
Corresponding Author: Dr. Jayakrishnan $C$.

Resident,

Department of Pathology,

Government Medical College,

Thrissur, Kerala, India.

E-mail:jcjkrish@gmail.com

DOI: $10.14260 /$ jemds/2020/64

Financial or Other Competing Interests: None.

How to Cite This Article:

Jayakrishnan C, Kamala VV. A comparative study of bronchial washing and brushing, with bronchial biopsy. J. Evolution Med. Dent. Sci. 2020;9(05):284-288, DOI: $10.14260 / \mathrm{jemds} / 2020 / 64$

Submission 02-11-2019,

Peer Review 09-01-2020

Acceptance 16-01-2020,

Published 03-02-2020.






\section{BACKGROUND}

Incidence and mortality attributed to lung cancer has risen steadily since the 1930 s, predominantly due to the popularity of cigarette smoking.(1) Today Lung cancer is the leading cause of cancer related mortality, both in the United States and worldwide. GBD 2015 estimated 18.6 million (18.0-19.4 million) new cases of cancer in 2015.(2) In India, According to a three year report (from 2006 to 2008) of population based cancer registries at Bangalore, Bhopal, Chennai, Delhi, and Mumbai, lung cancer was the leading site of cancer among males.(3) Early diagnosis, identification of the different histological types and of prognostic factors are valuable and which helps to direct patients for receiving appropriate treatment, in addition to identifying tumours at high chance of recurrence. As the global burden of this disease is much bigger, the diagnostic techniques which provide early detection and reliability are needed. In developing countries like India, cost effectiveness also plays an important role in the acceptance of such a test. Diagnostic cytology has now been widely accepted in all around the world as a reliable and cost-effective laboratory procedure. Bronchial cytology is a simple and informative method of early diagnosis of lung cancer and has raised a lot of interest.

Experience with the variety of sampling and preparation methods is important for cytologic interpretation because cytomorphology is different depending on the sampling and preparation method. The accuracy of respiratory cytology also varies according to the specimen type. Universal screening for lung cancer with chest radiographs and sputum cytology are not cost effective.(4) The use of low-dose spiral computed tomography has shown variable test results.(5) Detection of epigenetic or genetic aberrations in frequently mutated lung cancer genes (p53, K-ras, p16) from sputum samples show promises but which are not widely applicable in a cost effective manner.(6) Recently sputum sampling as the mainstay in evaluating respiratory cytology has declined significantly because of the advent of bronchoscopy. It is also provided improvement in sampling cells from the lower respiratory tract with the development of the flexible bronchoscope in the late 1960s. Today any part of the respiratory tract can be sampled with the help of a bronchoscope and its role in the diagnosis of lung diseases is unremarkable.

The role of bronchial washings and brushings under bronchoscopic control for the cytological diagnosis of lung cancer has been demonstrated by various studies. Since it is a cost effective and reliable way of screening for early lung cancer it can be widely applied especially in developing countries. Currently bronchial washing and brushing studies are routinely employed for the diagnosis of pulmonary neoplasm in different parts of the globe as well as in India. This study aims to assess the overall diagnostic yield of bronchial washing and brushing cytology compared bronchial biopsy in the diagnosis of bronchogenic carcinoma in our setting.

\section{Objectives}

1. To assess he overall diagnostic yield of bronchial washing and brushing cytology compared to bronchial biopsy, in bronchogenic carcinoma in our setting.
2. To determine the relative frequency of various cytological types of bronchogenic carcinoma diagnosed by these procedures in our population.

This evaluate the overall diagnostic yield of bronchial washing and brushing cytology compared to bronchial biopsy, in bronchogenic carcinoma in our setting. It will also provide the relative incidence of various cytological types of bronchogenic carcinoma diagnosed by these procedures in our population.

\section{METHODS}

This is a comparative cross-sectional study conducted in the Department of Pathology, Government Medical College, Thrissur from 1-1-2017 to 30-6-2018. 82 samples were included in the study. Sample size was taken based on the convenience of the study. Slides are examined under the microscope first with low power objectives (10X) and then under the high-power objectives $(40 \mathrm{X})$ to confirm the cytological features. Specimens that showed malignant characteristics will be classified as positive, while those with appearances suggestive but not diagnostic of malignancy will be classified as negative. Data obtained was analysed using software SPSS version 16.0.

\section{Inclusion Criteria}

Bronchial washing and brushing specimens received along with biopsy in the Department of Pathology, Government Medical College, Thrissur during the study period, which are suspicious for bronchogenic carcinoma. The suspicion for bronchogenic carcinoma is based on clinical or radiological features.

\section{Exclusion Criteria}

1. Bronchial wash or brushing specimen received without biopsy specimen.

2. Samples which are inadequate to prepare a cellular smear.

3. Bronchial biopsy specimens for non-malignant lung pathology, non-epithelial tumours, metastatic tumours and resected lung specimens were excluded from study.

\section{Methodology}

Cytological and histological specimens will be obtained by fibreoptic bronchoscopy under local anaesthesia. In bronchial washing sterile isotonic saline introduced into the bronchi bronchoscopically and washings from different bronchopulmonary segments are reaspirated, then smears are made from centrifuged deposits. Few slides will be air dried and fixed in $100 \%$ methanol for May-Grunwald Giemsa (MGG) staining. Few slides will be immediately fixed in a $95 \%$ ethanol for Papanicolaou's staining. Bronchial brushing can be done following washings. The area of suspected malignancy will be brushed two or three times; smears will be immediately fixed in 95\% alcohol and stained by Papanicolaou's method. Then a single specimen will be taken for histological examination from the same area by forceps and stained with haematoxylin and eosin after being processed. 
The slides will be examined under the microscope first with low power objectives (10X) and then the areas in the slides having cells will be focused under the high-power objectives (40X) to confirm the cytological features. Specimens that showed malignant characteristics will be classified as positive, while those with appearances suggestive but not diagnostic of malignancy will be classified as negative.

\section{Data Analysis}

Data were collected and entered in Microsoft office Excel 2010 sheet. Statistical analysis was done using SPSS software, version 16. Mean, median, and SD are used to describe quantitative data. Qualitative data are summarized using frequency and percentage.

\section{RESULTS}

\begin{tabular}{|c|c|c|}
\hline Age Group & Frequency & Percentage \\
\hline $20-39$ & 1 & 1.2 \\
\hline $40-59$ & 17 & 20.7 \\
\hline $60-79$ & 62 & 75.6 \\
\hline $80-99$ & 2 & 2.4 \\
\hline Total & $\mathbf{8 2}$ & $\mathbf{1 0 0}$ \\
\hline Sex & Fge Distribution of Carcinoma Lung Patients \\
\hline Males & 72 & Percentage \\
\hline Females & 10 & 87.8 \\
\hline \multicolumn{2}{|c|}{ Sex Distribution of Carcinoma Lung Patients } \\
\hline Table 1. Age and Sex Distribution of Carcinoma Lung Patients \\
\hline
\end{tabular}

\begin{tabular}{|c|c|c|}
\hline Smoking & Frequency & Percentage \\
\hline Present & 55 & 67.1 \\
\hline Absent & 27 & 32.9 \\
\hline Total & $\mathbf{8 2}$ & $\mathbf{1 0 0 . 0}$ \\
\hline \multicolumn{3}{|c|}{ Association of Smoking } \\
\hline Tumor Type & Frequency & Percentage \\
\hline Squamous cell carcinoma & 46 & 56 \\
\hline Adenocarcinoma & 14 & 17 \\
\hline Non-small cell carcinoma-nos & 15 & 18.3 \\
\hline Small cell carcinoma & 7 & 8.6 \\
\hline Total Tumour Types in Carcinoma Lung \\
\hline \multicolumn{3}{|c|}{ Table 2. Association of Smoking and Tumour Types in Carcinoma Lung } \\
\hline \multicolumn{3}{|c}{}
\end{tabular}

\begin{tabular}{|c|c|c|}
\hline Brushing & Frequency & Percent \\
\hline Positive & 29 & 35.4 \\
\hline Negative & 53 & 64.6 \\
\hline Total & $\mathbf{8 2}$ & $\mathbf{1 0 0 . 0}$ \\
\hline \multicolumn{3}{|c|}{ Positivity Rates of Bronchial Brushing } \\
\hline Washing & Frequency & Percent \\
\hline Positive & 6 & 7.3 \\
\hline Negative & 76 & 92.7 \\
\hline Total Positivity Rates of Bronchial Washing \\
\hline \multicolumn{3}{|c|}{ Table 3. Positivity Rates of Bronchial Brushing and Washing } \\
\hline
\end{tabular}

\begin{tabular}{|c|c|c|}
\hline Brush + Wash & Frequency & Percent \\
\hline Positive & 30 & 36.6 \\
\hline Negative & 52 & 63.4 \\
\hline Total & $\mathbf{8 2}$ & $\mathbf{1 0 0 . 0}$ \\
\hline \multicolumn{2}{|c|}{ Table 4. Positivity Rates When Bronchial Brushing and } \\
Washing were Combined \\
\hline
\end{tabular}

\begin{tabular}{|c|c|c|c|}
\hline SCC & Brushing & Washing & Combined \\
\hline Sensitivity & 19.6 & 4.3 & 19.6 \\
\hline Specificity & 100 & 100 & 97.2 \\
\hline PPV & 100 & 100 & 90 \\
\hline NPV & 49.4 & 45 & 43.9 \\
\hline Accuracy & 54.9 & 46.4 & 53.7 \\
\hline p value & 0.004 & 0.501 & 0.013 \\
\hline \multicolumn{4}{|c|}{ Table 5. Various Parameters in SCC Using Brushing, } \\
Washing and 'Combined' \\
\hline
\end{tabular}

82 bronchial biopsy specimens with a diagnosis of Carcinoma lung were studied. The age of the patients ranged from 35-84 years with a mean age of 64.14 years. Majority of patients were in the age group $60-79(75.6 \%)$. Out of the total 82 patients in this study there were 72 males and 10 females. Out of 82 patients, smoking was present in 55 patients $(67.1 \%)$. All of them were males and out of the 82 cases, 46 cases $(56 \%)$ were Squamous cell carcinoma, 14 cases $(17 \%)$ were Adenocarcinoma, 15 cases $(18.3 \%)$ were Non-small cell carcinoma-NOS, 7 cases $(8.6 \%)$ were Small cell carcinoma. In cases of Squamous cell carcinoma Bronchial brushing revealed sensitivity $-19.6 \%$, specificity $-100 \%$, positive predictive value $-100 \%$, negative predictive value$49.4 \%$, and accuracy-54.9\%. The values given by Bronchial washing was lower when 37 compared to Bronchial brushing, and the use of combination of both techniques provided no additive effects.

\section{DISCUSSION}

In the present study 82 patients, clinically suspected for carcinoma lung and had a positive report on bronchial biopsy are evaluated with bronchial brush and bronchial wash cytology, especially to determine the overall diagnostic yield of cytological techniques compared to histological diagnosis. Since the magnitude of carcinoma lung among various populations are still rising and the deadliest risk factor of cigarette smoking is still persisting, the control of the mortality due to carcinoma lung is possible only through early detection, advanced histological classification and specific treatment. Cytological techniques are getting more relevant especially with the bronchoscopic techniques and is a cost-effective screening method in developing countries like India. In this study age of the patients ranged from 35-84 years with a mean age of 64.14 years, and majority of patients were observed to be within the age group 60-79 (75.6\%) which is comparable to the latest Surveillance, Epidemiology and End

Results (SEER) data.(7) Lung cancer incidence for all subtypes is highest among those aged 75 years or older.(7) A study by Thomas VD et al in a tertiary care centre located in the same region (Thrissur, Kerala) also shows a similar mean age for the lung cancer patients.(8) Studies based on Indian population as observed by Noronha et al who conducted the study among 489 patients showed a median age as 56 years,(9) which was slightly lower when compared with the present study. This may be due to the small sample size of the present study. Out of the total 82 patients included in this study, $87.8 \%$ were males and $12.2 \%$ were females. This was comparable to the studies by Anupam Sarma et al. According his study among 69 patients $82.61 \%$ were male and $17.39 \%$ females, the M: F ratio being 4.75:1.(10) In the present study $\mathrm{M}: \mathrm{F}$ ratio was $7.2: 1$. Smoking was associated with $67.1 \%$ of the cases, all of them were males. Smoking tobacco remains as the single most important risk factor. Western studies show increased incidence of lung cancer among females when compared to Indian trends, this is probably due to the change in the cultural and social lifestyle. Risk of smoking among males and females are found to be equal in western population.(11) This was evidenced by recent meta-analysis by 
O'Keeffe LM, et al. Out of the total 82 cases included in this study, it was observed that 46 cases (56\%) were Squamous cell carcinoma, 14 cases (17\%) were Adenocarcinoma, 7 cases $(8.6 \%)$ were Small cell carcinoma, 15 cases $(18.3 \%)$ were Non-small cell carcinoma-NOS.

Western literature says prior to 1970 s, squamous cell carcinoma was the most common histological type of NSCLC. However, thereafter dramatic increase in adenocarcinoma is observed, making it as the predominant histological subtype of NSCLC.(9) Findings from our study was concordant with studies by Singh N et al.(8) Another review article by Behera et al in 2004 also showed squamous cell carcinoma as the predominant subtype in India.(3) This changes may be due to lack of adequate studies in Indian population and demographic differences. However, a recent study in India by Krishnamurthy $\mathrm{A}$ et al shows a changing trend towards adenocarcinoma.(10) In the present study bronchial brushing and bronchial washing results of 82 patients with biopsy proven malignancy are analysed. Out of 82 patients bronchial brushing was positive in 29 cases $(35.4 \%)$ regardless of tumour types. Here findings suggestive/suspicious of malignancy were taken as 'Negative' in order to know the definite positivity rate. Analysis also done with considerations by taking findings suggestive/suspicious of malignancy taken as a sign of possible malignancy. 43 out of 82 cases $(52.4 \%)$ were positive in such analysis. The diagnostic sensitivity of Bronchial brushing in the diagnosis of lung cancer is variable in different studies from 48$85 \% .(12,13)$ This wide variation can be explained by different techniques used to obtain the cytological samples and also inclusion of suspicious/suggestive cases as positive when calculating the sensitivity. In our study the sensitivity of bronchial brushing regardless of tumour subtypes was $35.4 \%$. Inclusion of suspicious cases as positive yielded an overall sensitivity of $52.4 \%$. This was comparable to the study by Liam et al, their study among 503 patients with confirmed lung cancer showed a sensitivity of bronchial brushing as $53.7 \%$. Bronchial washing was able to diagnose only 6 cases out of 82 (7.3\%), which was increased to 16 cases $(19.5 \%)$ when the findings suggestive/suspicious of malignancy also taken into account. Present study showed lower positivity rate of bronchial washing compared to bronchial brushing. Different Studies by Mak VH et al and Wong PC et al showed similar sensitivity for washing and brushing. Various studies showed lower sensitivity of bronchial wash compared to bronchial brushing. $(14,15,16)$ Liam et al also showed lower sensitivity of bronchial washing as $28.3 \%$ which was comparable to our studies.(14)

Bodh $\mathrm{A}$ et al showed increase in overall sensitivity if both bronchial brushing and washing are used together. Analysis also done by combining both cytological techniques, that is bronchial brushing and washing together. Definite Positivity of bronchial washing and/or brushing was taken as positive for malignancy, 30 cases out of 82 (36.6\%) were positive. When findings suggestive/suspicious of malignancy also considered, combination of both cytological techniques yielded 45 positive cases out of 82 (54.9\%). In our study addition of bronchial washing into bronchial brushing increased overall sensitivity by $2.5 \%$. A similar value was obtained in a study by Ruchee Khandelwal et al, in which addition of bronchial wash and brush increased positive yield by only $2.2 \%$. Analysis of bronchial brushing and washing done in different major histological subtypes, such as Squamous cell carcinoma, Adenocarcinoma, NSCLC-NOS, and Small cell carcinoma.

\section{Bronchial Brushing, Washing and Combined in Biopsy Proven Squamous Cell Carcinoma}

Out of 82 cases, 46 cases were biopsy proven Squamous cell carcinoma. Out of these 46 cases bronchial brushing given positivity in 9 cases, bronchial washing in 2 cases only. When both combined given positivity in 9 cases. In case of Squamous cell carcinoma, bronchial brushing alone detected more number of cases compared to bronchial washing alone and when both cytological techniques were combined, there was no additive usefulness because of bronchial washing. In cases of Squamous cell carcinoma Bronchial brushing revealed sensitivity $-19.6 \%$, specificity $-100 \%$, positive predictive value $-100 \%$, negative predictive value- $49.4 \%$, and accuracy-54.9\%. Bronchial washing revealed a lower sensitivity $4.3 \%$ and accuracy $46.4 \%$ when both techniques combined there was no additive effects of bronchial washing over brushing.

\section{Bronchial Brushing, Washing and Combined in Biopsy Proven Adenocarcinoma}

Out of 82 cases, 14 cases were biopsy proven Adenocarcinoma. Out of these 14 cases bronchial brushing given positivity in 4 cases, bronchial washing given positivity in 1 case and when both combined given positivity in 5 cases. In case of Adenocarcinoma, bronchial brushing alone detected more number of cases compared to bronchial washing alone but, when both cytological techniques were combined, there was additive usefulness of bronchial washing. In cases of Adenocarcinoma, Bronchial brushing revealed sensitivity $-28.6 \%$, specificity $-92.6 \%$, PPV- $44.4 \%$, NPV-82.9\%, and accuracy-81.7\%. Bronchial washing revealed sensitivity $7.1 \%$, specificity $-98.5 \%$, PPV-50\%, NPV-83.7\%, and accuracy-82.9\%. When both techniques combined had a sensitivity of $35.7 \%$, specificity $92.6 \%$, PPV- 50\%, NPV $87.5 \%$ and accuracy $82.9 \%$. Adenocarcinoma cases show valuable use of bronchial washing along with bronchial brushing for a better yield in the diagnosis by increasing the sensitivity, specificity and accuracy.

\section{Bronchial Brushing, Washing and Combined in Biopsy Proven Non-Small Cell Lung Carcinoma (NSCLC)-Nos}

Out of 82 cases, 15 cases were biopsy proven Non-Small cell lung carcinoma (NSCLC)-Nos. Out of these 15 cases bronchial brushing given positivity in 4 cases, bronchial washing given positivity in 2 cases and when both combined given positivity in 4 cases. In case of NSCLC-Nos, bronchial brushing alone detected more number of cases compared to bronchial washing alone and when both cytological techniques were combined, there was no additive usefulness of bronchial washing. In cases of NSCLC-Nos Bronchial brushing revealed sensitivity $-26.7 \%$, specificity $-91 \%$, PPV-40\%, NPV-84.7\%, and accuracy-79.3\%. The values given by Bronchial washing was lower when compared to Bronchial brushing, and the usefulness of combination of both techniques provided no additive 
Bronchial Brushing, Washing and Combined in Biopsy Proven Small Cell Lung Carcinoma (SCLC)

Out of 82 cases, 7 cases were biopsy proven Small cell lung carcinoma (SCLC). Out of these 7 cases bronchial brushing given positivity in 2 cases, bronchial washing given positivity in 2 cases and when both combined given positivity in 2 cases. In cases of Small cell lung carcinoma, Bronchial brushing, bronchial washing and when both combined revealed similar values. Sensitivity $-28.6 \%$, specificity - 100\%, positive predictive value $-100 \%$, negative predictive value93.8\%, and accuracy-94\%.

In summary bronchial brushing has better sensitivity among different subtypes of carcinoma lung. Even though various studies show usefulness of bronchial washing either alone or in combination with bronchial brushing, in our study additive effect of washing was observed in adenocarcinoma cases only.

\section{CONCLUSIONS}

Among routinely employed cytological techniques in our centre for the diagnosis of bronchogenic carcinoma, bronchial brushing has higher sensitivity and specificity than bronchial washing. Even though combination of both cytological techniques yielded not much difference in sensitivity and specificity than brushing alone, usefulness of bronchial washing was evident in adenocarcinomas. Therefore, bronchial brushing, washing and biopsy should be done in the evaluation of suspicious bronchogenic carcinoma and major importance in the processing, evaluation and analysis of brushing will be useful in better cytological diagnosis.

\section{REFERENCES}

[1] Hammond EC, Horn D. Smoking and death rates-report on forty-four months of follow up of 187,783 men. J Am Med Assoc 1958;166(11):1294-308.

[2] GBD 2015 Disease and Injury Incidence and Prevalence Collaborators. Global, regional and national incidence, prevalence and years lived with disability for 310 diseases and injuries, 1990-2015: a systematic analysis for the Global Burden of Disease Study 2015. Lancet 2016;388(10053):1545-602.

[3] Behera D, Balamugesh T. Lung Cancer in India. Indian J Chest Dis Allied Sci 2004;46(4):269-81.
[4] Bach PB, Jett JR, Pastorino U, et al. Computed tomography screening and lung cancer outcomes. JAMA 2007;297(9):953-61.

[5] Fumagalli C, Bianchi F, Raviele PR, et al. Circulating and tissue biomarkers in early-stage non-small cell lung cancer. Ecancermedicalscience 2017;11:717.

[6] Singh N, Aggarwal AN, Gupta D, et al. Unchanging clinicoepidemiological profile of lung cancer in north India over three decades. Cancer Epidemiol 2010;34(1):101-4.

[7] Noone AM, Cronin KA, Altekruse SF, et al. Cancer incidence and survival trends by subtype using data from the surveillance epidemiology and end results program, 1992-2013. Cancer Epidemiol Biomarkers Prev 2017;26(4):632-41.

[8] Thomas VD, Jose B, Rennis DK, et al. Prevalence of type and etiology of lung cancer among the patients presented to a tertiary care hospital at central Kerala: a descriptive study. Int J Res Med Sci 2018;6(3):834-7.

[9] Noronha V, Dikshit R, Raut N, et al. Epidemiology of lung cancer in India: focus on the differences between nonsmokers and smokers: a single-centre experience. Indian Journal of Cancer 2012;49(1):74-81.

[10] Sarma A, Sharma JD, Bhuyan C, et al. A study of cytological evaluation of bronchial washing and brushing in bronchogenic carcinoma. IJSRP 2013;3(8):1-7.

[11] O'Keeffe LM, Taylor G, Huxkey RR, et al. Smoking as a risk factor for lung cancer in women and men: a systematic review and meta-analysis. BMJ Open 2018;8(10):e021611.

[12] Rekhtman N, Brandt SM, Sigel CS, et al. Suitability of thoracic cytology for new therapeutic paradigms in nonsmall cell lung carcinoma: high accuracy of tumor subtyping and feasibility of EGFR and KRAS molecular testing. J Thorac Oncol 2011;6(3):451-8.

[13] Tuladhar A, Panth R, Joshi A. Comparative analyses of cytohistologic techniques in diagnoses of lung lesions. J Pathol Nepal 2011;1:126-30.

[14] Liam CK, Pang YK, Poosparajah S. Diagnostic yield of flexible bronchoscopic procedures in lung cancer patients according to tumour location. Singapore Med J 2007;48(7):625-31.

[15] Dasgupta A, Jain P, Minai OA, et al. Utility of transbronchial needle aspiration in the diagnosis of endobronchial lesions. Chest 1999;115(5):1237-41.

[16] Solomon DA, Solliday NH, Gracey DR. Cytology in fiberoptic bronchoscopy: comparison of bronchial brushing, washing and post-bronchoscopy sputum. Chest 1974;65(6):616-9. 